\title{
Calibrating the In Vitro-In Vivo Correlation for OATP-Mediated Drug-Drug Interactions with Rosuvastatin Using Static and PBPK Models $^{\circledR}$
}

\author{
Rucha Sane, ${ }^{\circ}$ Kit Wun Kathy Cheung, Péter Kovács, Taleah Farasyn, ${ }^{1}$ Ruina Li, Annamaria Bui, \\ (1) Luna Musib, Emese Kis, Emile Plise, and Zsuzsanna Gáborik
}

Departments of Clinical Pharmacology (R.S., K.W.K.C., T.F., L.M.) and Drug Metabolism and Pharmacokinetics (T.F., R.L., E.P.), Genentech, Inc., South San Francisco, California; and SOLVO Biotechnology, Budapest, Hungary (P.K., A.B., E.K., Z.G.)

Received June 15, 2020; accepted September 28, 2020

\section{ABSTRACT}

Organic anion-transporting polypeptide (OATP) 1B1/3-mediated drug-drug interaction (DDI) potential is evaluated in vivo with rosuvastatin (RST) as a probe substrate in clinical studies. We calibrated our assay with RST and estradiol 17- $\beta$-D-glucuronide $\left(E_{2} 17 \beta G\right) / c h o l e c y s t o k i n i n-8(C C K 8)$ as in vitro probes for qualitative and quantitative prediction of OATP1B-mediated DDI potential for RST. In vitro OATP1B1/1B3 inhibition using $E_{2} 17 \beta G$ and CCK8 yielded higher area under the curve (AUC) ratio (AUCR) values numerically with the static model, but all probes performed similarly from a qualitative cutoff-based prediction, as described in regulatory guidances. However, the magnitudes of DDI were not captured satisfactorily. Considering that clearance of RST is also mediated by gut breast cancer resistance protein (BCRP), inhibition of BCRP was also incorporated in the DDI prediction if the gut inhibitor concentrations were $10 \times I_{50}$ for BCRP inhibition. This combined static model closely predicted the magnitude of RST DDI with root-meansquare error values of 0.767-0.812 and 1.24-1.31 with and without BCRP inhibition, respectively, for in vitro-in vivo correlation of DDI. Physiologically based pharmacokinetic (PBPK) modeling was also used to simulate DDI between RST and rifampicin, asunaprevir, and velpatasvir. Predicted AUCR for rifampicin and asunaprevir was within 1.5-fold of that observed, whereas that for velpatasvir showed a 2-fold underprediction. Overall, the combined static model incorporating both OATP1B and BCRP inhibition provides a quick and simple mathematical approach to quantitatively predict the magnitude of transporter-mediated DDI for RST for routine application. PBPK complements the static model and provides a framework for studying molecules when a dynamic model is needed.

\section{SIGNIFICANCE STATEMENT}

Using 22 drugs, we show that a static model for organic anion-transporting polypeptide (OATP) 1B1/1B3 inhibition can qualitatively predict potential for drug-drug interaction (DDI) using a cutoff-based approach, as in regulatory guidances. However, consideration of both OATP1B1/ 3 and gut breast cancer resistance protein inhibition provided a better prediction of the magnitude of the transporter-mediated DDI of these inhibitors with rosuvastatin. Based on these results, we have proposed an empirical mechanistic-static approach for a more reliable prediction of transporter-mediated DDI liability with rosuvastatin that drug development teams can leverage.
There was no external funding for this work.

${ }^{1}$ Former affiliation.

Financial disclosure: R.S., K.W.K.C., R.L., L.M., T.F., and E.P. were employees of Genentech, a member of the Roche Group, when the work was performed. The employees are also holders of Roche Holding Ltd. stock. P.K., A.B., E.K., and Z.G. were employees of SOLVO Biotechnology, a Charles River Company, when the work was performed.

https://doi.org/10.1124/dmd.120.000149.

S This article has supplemental material available at dmd.aspetjournals.org.
Introduction

Organic anion-transporting polypeptides (OATPs) 1B1/3, which are mainly expressed in the liver, are clinically important transporters for drug-drug interactions (DDIs) (https://www.ema.europa.eu/documents/ scientific-guideline/guideline-investigation-drug-interactions_en.pdf; https://www.pmda.go.jp/files/000228122.pdf; https://www.fda.gov/ media/134582/download). During drug development, the in vivo OATP1B-mediated DDI liability of an investigational drug is often assessed using in vitro inhibition assays, and the in vitro inhibition data are used to predict the potential to inhibit these transporters in vivo.

ABBREVIATIONS: AUC, area under the curve; AUCR, AUC ratio; BCRP, breast cancer resistance protein; CCK8, cholecystokinin-8; DDI, drug-drug interaction; $\mathrm{E}_{2} 17 \beta \mathrm{G}$, estradiol 17- $\beta$-D-glucuronide; FN, false negative; FP, false positive; HEK293, human embryonic kidney 293 ; Igut, intestinal luminal concentration of the inhibitor; MRM, Multiple Reaction Monitoring; NPV, negative predictive value; NTCP, sodium-taurocholate cotransporting polypeptide; OATP, organic anion-transporting polypeptide; PBPK, physiologically based pharmacokinetic; PPV, positive predictive value; RMSE, root-mean-square error; RST, rosuvastatin; TN, true negative; TP, true positive. 
Since inhibition can be substrate-dependent, careful choice of in vitro probes is an essential first step to minimize false negative (FN) as well as false positive (FP) predictions (Izumi et al., 2015; https://www.fda. gov/media/134582/download). Prototypical in vitro probe substrates for OATP1B1 and OATP1B3 include estradiol 17- $\beta$-D-glucuronide $\left(\mathrm{E}_{2} 17 \beta \mathrm{G}\right)$ and cholecystokinin-8 (CCK8) (Izumi et al., 2015). One of the frequently used in vivo probe substrates of OATP1B1/3 DDI studies is rosuvastatin (RST) (Izumi et al., 2015; https://www.fda.gov/ drugs/drug-interactions-labeling/drug-development-and-drug-interactionstable-substrates-inhibitors-and-inducers).

The science of in vitro to in vivo extrapolation for transporter-related DDI encompasses several perspectives and considerations. For instance, for a qualitative prediction of whether an in vitro inhibitor of OATP1B1/ 3 is likely to inhibit these transporters in vivo, the regulatory guidances have recommended cutoff values for $R$ (the predicted ratio of the area under the curve in the presence and absence of the investigational drug as an inhibitor) based on a basic model (https://www.ema.europa.eu/ documents/scientific-guideline/guideline-investigation-drug-interactions_en. pdf; https://www.pmda.go.jp/files/000228122.pdf; https://www.fda. gov/media/134582/download). These DDI guidances highlight the need to eliminate false negatives and propose an empirical qualitative cutoff value-based assessment to determine whether in vivo DDI is likely. From a clinical pharmacology perspective, a quantitative prediction is of high relevance to understand clinical impact of a DDI. To that end, mechanistic models have been applied for DDI prediction.

For success of either a cutoff-based approach or a mechanistic model-based prediction, appropriate in vitro data that give the most accurate predictions are vital to provide optimal guidance to the drug development teams as well as patients enrolled in the studies. We evaluated the predictive performance of RST versus $E_{2} 17 \beta \mathrm{G}$ and CCK8 in our in vitro inhibition assay for qualitative $R$-value cutoff-based prediction as well as quantitative prediction of DDI using 22 compounds. We further attempted to holistically approach transporter DDI prediction for these 22 compounds by using a combined static mechanistic model that takes into consideration in vitro inhibition of OATP1B1/3 and breast cancer resistance protein (BCRP), based on the mechanistic information about RST that its clearance is mediated by both OATP1B1/3 and gut BCRP. Lastly, we also used physiologically based pharmacokinetic (PBPK) modeling to simulate the DDI between RST and 3 of the 22 studied drugs, rifampicin, asunaprevir, and velpatasvir, to evaluate the DDI potential of these drugs using the dynamic method to evaluate whether PBPK would further refine the predictions beyond the other approaches.

\section{Materials and Methods}

\section{Source of Substrates and Inhibitors}

RST, $\mathrm{E}_{2} 17 \beta \mathrm{G}$, and CCK8 (Sigma) were used as substrates in this study. For radiolabeled tracing, $\left[{ }^{3} \mathrm{H}\right]-\mathrm{E}_{2} 17 \beta \mathrm{G}$ and $\left[{ }^{3} \mathrm{H}\right]-\mathrm{CCK} 8$ were used, which were both purchased from PerkinElmer Life and Analytical Sciences. OATP1B inhibitory potential of 22 drugs was investigated. Sixteen of these 22 drugs (i.e., baicalin, cyclosporine A, darunavir, digoxin, erythromycin, ezetimibe, fluconazole, gemfibrozil, grazoprevir, ketoconazole, lopinavir, metformin, rifampicin, ritonavir, telmisartan, and valsartan) were purchased from Sigma; three (i.e., atazanavir, fimasartan, and velpatasvir) were purchased from Selleckchem; and asunaprevir, eltrombopag, and eluxadoline were purchased from Carbosynth, SeqChem, and Toronto Research Chemicals, respectively.

\section{Identification of Drugs that Have Been Studied in Clinical DDI Studies with RST}

Using the University of Washington DDI Database (https://www. druginteractioninfo.org), 22 drugs that were previously investigated and coadministered with RST in clinical DDI studies were identified (Supplemental Table 1). Here, we defined a drug to be an inhibitor of OATP1B if the observed area under the curve ratio (AUCR) of the substrate was $\geq 1.25$, which was consistent with the definition in previous studies (Yoshida et al., 2012; Vaidyanathan et al., 2016). Twelve of these 22 drugs (asunaprevir, eltrombopag, grazoprevir, lopinavir, ritonavir, velpatasvir, atazanavir, cyclosporine, darunavir, eluxadoline, gemfibrozil, and rifampicin) showed observed AUCR $\geq 1.25$, and 10 of them (baicalin, ezetimibe, ketoconazole, telmisartan, digoxin, erythromycin, fimasartan, fluconazole, metformin, and valsartan) had observed AUCR $<1.25$ (Supplemental Table 1). OATP inhibition of these 22 drugs was assessed in vitro using $\mathrm{E}_{2} 17 \beta \mathrm{G}$ (for OATP1B1), CCK8 (for OATP1B3), and RST (for both OATP1B1 and OATP1B3) as probe substrates (Supplemental Table 1).

\section{Cell System and Transporter Inhibition Assay for OATP1B1/3}

Transporter inhibition assay was conducted as follows. Briefly, human embryonic kidney 293 (HEK293) cells overexpressing OATP1B1 or OATP1B3 were maintained in Dulbecco's modified Eagle's medium supplemented with $10 \%$ fetal bovine serum, $100 \mathrm{U} / \mathrm{ml}$ penicillin, $100 \mathrm{U} / \mathrm{ml}$ streptomycin, and $100 \mu \mathrm{g} / \mathrm{ml}$ hygromycin B. Cells were seeded at a density of 100,000 cells per well in polyD-lysine-coated 96-well plates 12-18 hours before the inhibition experiments. Cells were first preincubated for 30 minutes in Hank's balanced salt solution (HBSS) containing 1 of the 22 tested drugs at increasing concentration, and this was followed by coincubation with the same inhibitor and RST $(0.1$ and $1 \mu \mathrm{M}$ for OATP1B1 and OATP1B3, respectively) for 1 minute. In the case of ${ }^{3} \mathrm{H}-\mathrm{E}_{2} 17 \beta \mathrm{G}$ and ${ }^{3} \mathrm{H}$-CCK8, $1 \mu \mathrm{M}$ for 3 minutes and $1 \mu \mathrm{M}$ for 2 minutes were used, respectively, traced with radioactive substrate. In the case of ${ }^{3} \mathrm{H}-\mathrm{E}_{2} 17 \beta \mathrm{G}$ and ${ }^{3} \mathrm{H}$-CCK8 measurements, cells were lysed with $0.1 \mathrm{M} \mathrm{NaOH}$ and then analyzed by liquid scintillation. For the samples analyzed by mass spectrometry (RST), cells were washed twice with ice-cold HBSS and then lysed with 35\% methanol/ $25 \%$ acetonitrile containing $20 \mathrm{nM}$ RST-d6 internal standard. This analysis was carried out with a Shimadzu Nexera UPLC system coupled to a QTRAP 5500 AB Sciex in positive ion mode. Mobile phase A was water with $0.1 \%$ formic acid, and B was acetonitrile with $0.1 \%$ formic acid. The chromatography was performed on a Phenomenex Kinetex column $2.6 \mu \mathrm{m}$ XB-C18 $50 \times 2.1 \mathrm{~mm}$ (Torrance, CA). The gradient was started with $10 \% \mathrm{~B}$ and then increased to $90 \% \mathrm{~B}$ in 0.6 minutes and maintained at $90 \% \mathrm{~B}$ for another 0.2 minutes, decreased to $10 \% \mathrm{~B}$ within 0.01 minutes, and maintained at $10 \% \mathrm{~B}$ for another 0.2 minutes. The flow rate was $1.2 \mathrm{ml} / \mathrm{min}$, and the cycle time (injection to injection including instrument delays) was approximately 1.0 minute. The sample injection volume was $3 \mu$. Multiple Reaction Monitoring transition was $482.091 \rightarrow 258 / 2$ for RST and $488.2 \rightarrow 258.2$ for the RST-d6 as the internal standard. IC $_{50}$ values were then estimated.

\section{Vesicular Transport Assay for BCRP Inhibition}

BCRP inhibition data of the investigated drugs were collected from literature if available, as shown in Supplemental Table 2. In all other cases, the inhibitory effects of the perpetrators on transport of RST in membrane vesicles from HEK293 cells overexpressing human BCRP were investigated with rapid filtration techniques, as described previously (Heredi-Szabo et al., 2012). All experiments were performed in triplicate. $\mathrm{IC}_{50}$ values were then calculated using the specific transport data, which were acquired by subtracting transport measured in the absence of ATP, from transport measured in presence of ATP.

After the vesicular transport assay, the vesicles containing the RST on a filter plate were lysed with $67 \%$ methanol/33\% water solution and filtered by centrifugation at $3700 \mathrm{rpm}$. The bioanalysis was carried out with a Thermo Scientific Dionex UltiMate 3000 series UHPLC coupled to a Thermo Scientific TSQ Quantum Access Max Mass Spectrometer in positive ion mode. Mobile phase A was water with $0.1 \%$ formic acid, and B was acetonitrile with $0.1 \%$ formic acid. The chromatography was performed on a Poroshell 120 EC-C18 $3.0 \times 50 \mathrm{~mm} 2.7 \mu \mathrm{m}$ (Agilent Technologies, CA). The gradient was started with $40 \% \mathrm{~B}$ until 0.2 minutes and then increased to $95 \% \mathrm{~B}$ within 0.01 minutes and maintained at $95 \% \mathrm{~B}$ for another 0.59 minutes, decreased to $40 \% \mathrm{~B}$ within 0.01 minutes, and maintained at $40 \% \mathrm{~B}$ for another 1.19 minutes. The flow rate was $0.5 \mathrm{ml} / \mathrm{min}$, and the cycle time (injection to injection, including instrument delays) was approximately 3.2 minutes. Multiple Reaction Monitoring transition was $482.207 \rightarrow 258.180$ for RST. IC 50 values were then estimated. 


\section{$R$-Value Calculations Using Basic Model}

AUCR estimated by basic model was calculated using the following equation (https://www.fda.gov/media/134582/download):

$$
\begin{aligned}
R & =1+\frac{I_{u, i n, \max }}{I C_{50}} ; \text { where } I_{u, i n, \max } \\
& =f_{u, p} \times\left(\left(C_{\max }+\left(k_{a} \times \text { Dose } \times F_{a} F_{g}\right)\right) / Q_{h} / R_{B}\right)
\end{aligned}
$$

$I_{u, i n, m a x}$ is the estimated maximum unbound plasma inhibitor concentration at the inlet to the liver.

$f_{u, p}$ is the unbound fraction in plasma; for highly bound drugs, the $f_{u, p}$ were rounded up to 0.01 for this part of the calculation. (Supplemental Table 1) $\mathrm{C}_{\max }$ is the maximal plasma concentration.

$F_{a}$ is the fraction absorbed. $F_{a}=1$ was used as the worst-case estimate.

$F_{g}$ is the intestinal availability. $F_{g}=1$ was used as the worst-case estimate.

$\mathrm{k}_{\mathrm{a}}$ is the absorption rate constant. $\mathrm{ka}=0.1 / \mathrm{min}$ was used as the worst-case estimate.

$\mathrm{Q}_{\mathrm{h}}$ is the hepatic blood flow rate. $\mathrm{Q}_{\mathrm{h}}=1500 \mathrm{ml} / \mathrm{min}$.

$R_{B}$ is the blood-to-plasma concentration ratio.

$\mathrm{R}_{\mathrm{B}}$ was assumed to be 1 .

\section{Static Models for DDI Predictions}

The in vivo OATP1B1/3-mediated DDI magnitude with RST was quantitatively predicted using the following basic static model equation:

$$
\text { AUCR predicted by static model }=\left(\frac{1}{\sum \frac{f e, O A T P 1 B}{1+\frac{\left.\mid I_{\text {in, }, \text {, max }}\right]}{I C 50}}+\left(1-\sum f_{e, O A T P 1 B}\right)}\right)
$$

in which $\mathrm{f}_{\mathrm{e}, \mathrm{OATP} 1 \mathrm{~B}}$ is the fraction of systemic clearance of RST that was mediated by OATP1B1 and $1 \mathrm{~B} 3$.

$\mathrm{I}_{\mathrm{u}, \mathrm{in}, \max }$ is the estimated maximum unbound plasma inhibitor concentration at the inlet to the liver.

Since hepatic clearance contributes to $72 \%$ of the total body rosuvastatin clearance, and the relative contributions of OATP1B1, OATP1B3, and sodiumtaurocholate cotransporting polypeptide (NTCP) to the overall hepatic uptake of RST have been estimated to be $70 \%, 20 \%$, and $10 \%$, respectively (Elsby et al., 2012; Wang et al., 2017), the $\mathrm{f}_{\mathrm{e}, \text { OATP1B1 }}$ equals 0.504 , and $\mathrm{f}_{\mathrm{e}, \mathrm{OATP} 1 \mathrm{~B} 3}$ is approximately 0.144 .

For those studied drugs that had the potential to inhibit intestinal BCRP in vivo as determined by the criteria of $\mathrm{I}_{\text {gut }} / \mathrm{IC}_{50} \geq 10$, in which $\mathrm{I}_{\text {gut }}=$ dose of studied drug/250 ml (https://www.fda.gov/media/134582/download), eq. 2 was modified to predict the overall AUCR caused by both gut BCRP and OATP1B1/3 inhibition (combined static model):

AUCR predicted by combined static model $=$ AUCR caused by inhibition of BCRP in gut $\times$ AUCR caused by OATP1B1/3 inhibition

$$
=\left(\frac{1}{\frac{f_{e, B C R P}}{1+\frac{I_{\text {, } m a x}}{I C_{50}}}+\left(1-f_{e, B C R P}\right)}\right) \times\left(\frac{1}{\sum \frac{f e, \text { OATP1B }}{1+\frac{I_{\text {Iin, }, \text { max }}}{I C 50}}+\left(1-\sum f_{e, O A T P 1 B}\right)}\right)
$$

in which $\mathrm{I}_{\text {gut }}$ is the intestinal luminal concentration of the inhibitor, $\mathrm{f}_{\mathrm{e}, \mathrm{BCRP}}$ is the fraction excreted by BCRP in the gut, which is 0.5 (Elsby et al., 2016)(), and $\mathrm{f}_{\mathrm{e}, \text { OATPIB }}$ is the fraction of systemic clearance of RST that was mediated by OATP1B1 and OATP1B3.

\section{Model Performance Assessment for Static and Combined Static Model}

In addition to the U.S. Food and Drug Administration-recommended cutoff values of $R \geq 1.1$, the cutoff recommended by European Medicines Agency $(R \geq$ 1.04) was also used to calibrate the in vitro system (https://www.ema.europa.eu/ documents/scientific-guideline/guideline-investigation-drug-interactions_en.pdf; https://www.fda.gov/media/134582/download).

To qualitatively assess the predictive performance of RST and $E_{2} 17 \beta \mathrm{G} /$ CCK8 using cutoff-based approach, the number of true positive (TP), true negative (TN), FP, and FN were first tallied. The negative predictive value (NPV) and the positive predictive value (PPV) were calculated to evaluate the accuracy of the transporter-mediated DDI prediction with the studied probes:

$$
\begin{aligned}
\mathrm{NPV} & =\mathrm{TN} /(\mathrm{TN}+\mathrm{FN}) \\
\mathrm{PPV} & =\mathrm{TP} /(\mathrm{TP}+\mathrm{FP})
\end{aligned}
$$

To quantitatively assess the predictive performance of RST and $\mathrm{E}_{2} 17 \beta \mathrm{G} /$ CCK8, the root-mean-square error (RMSE) was calculated using the following equation:

$$
R M S E=\sqrt{\frac{1}{N} \sum(\text { Predicted }- \text { Observed })^{2}}
$$

\section{Retrospective Prediction of Clinical DDI with PBPK Modeling}

DDI simulation with RST using PBPK approach was performed for 3 of the 22 drugs. These three drugs were selected based on their OAT1B and intestinal BCRP inhibitory potencies: Rifampicin is a well-known strong OATP1B inhibitor, velpatasvir is a moderate OATP1B inhibitor, and asunaprevir is a weak OATP1B inhibitor.

Drug PBPK Model Establishment. All model files were established using SimCYP Simulator (v18; Certara Company, Sheffield, UK). The RST model was established based on the model by Wang et al. (2017) with slight modification in the Caco-2 permeability and the Kp (tissue: plasma partition coefficient) scalar used in distribution, as described in Supplemental Table 3. The drug-dependent parameters for rifampicin, velpatasvir, and asunaprevir were taken from the literature (Supplemental Table 4). To verify the individual drug models, visual check of the simulated concentration-time profiles and the predicted $\mathrm{C}_{\max }$ and AUC were compared with the observed data gathered from published studies. The model was considered acceptable if the observed profile was largely contained within the 5th and the 95th percentile of the predicted profile and the overall shape of the predicted pharmacokinetic (PK) profile was similar to that of the observed profile. For rosuvastatin PK in presence of inhibitors, if only AUC and $\mathrm{C}_{\max }$ changes are reported in the literature, then only those values were compared (Supplemental Figs. 1 and 2).

DDI Simulations. Clinical DDIs between the three drugs (rifampicin, asunaprevir, and velpatasvir) and RST were predicted using the established models. The model parameters are provided in Supplemental Table 5. Based on other published articles, it was expected that the $\mathrm{IC}_{50}$ values of the three drugs would need to be adjusted using a scaling factor to closely predict the observed clinical DDIs (Chen et al., 2018; Yoshida et al., 2018). The optimization of this scaling factor was an iterative "top-down" process during which various incremental scaling factors $(10 \times, 100 \times, 200 \times)$ were used, and substratespecific optimal scaling factor that was predictive of the DDI for all three inhibitors for the given substrates was selected. The simulated AUCRs were compared with those reported in clinical DDI studies (Prueksaritanont et al., 2014, 2017; Eley et al., 2015; Lai et al., 2016; Mogalian et al., 2016; Shen et al., 2017; Takehara et al., 2018)

Software. $\mathrm{IC}_{50}$ calculation and graphical exploration were performed using GraphPad Prism software (v8.1.1; La Jolla, CA). SimCYP Simulator v.18 (Certara Company) was used for all the PBPK modeling and simulation. All the PBPK simulations were performed with virtual adult populations of 100 virtual subjects.

\section{Results}

IC $_{50}$ Values Measured and AUCR Estimated by Basic Model. $\mathrm{IC}_{50}$ values of the 22 studied drugs measured in the in vitro inhibition assay are reported in Supplemental Table 1. In general, the $\mathrm{IC}_{50}$ values for OATP1B1 and OATP1B3 when RST was used as in vitro probe substrate were higher than those for $\mathrm{E}_{2} 17 \beta \mathrm{G}$ and CCK8.

Subsequently, in vitro assays using $\mathrm{E}_{2} 17 \beta \mathrm{G} / \mathrm{CCK} 8$ as in vitro probe substrates generally yielded higher $R$ values for majority of the 22 drugs tested than those using RST as in vitro probe substrate (Fig. 1; Supplemental Table 1). Exceptions include gemfibrozil (2.09 vs. 1.25 ), darunavir (1.68 vs. 1.37 ), and eluxadoline (1.27 vs. 1.05$)$ for $\mathrm{E}_{2} 17 \beta \mathrm{G}$ versus RST, respectively, for OATP1B1 and atazanavir (3.5 vs. 
OATP1B1 R-values

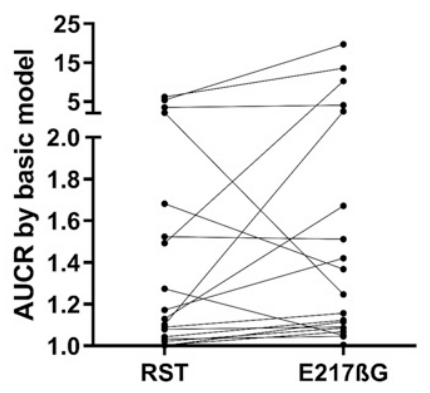

Probe substrates

\section{B OATP1B3 R-values}

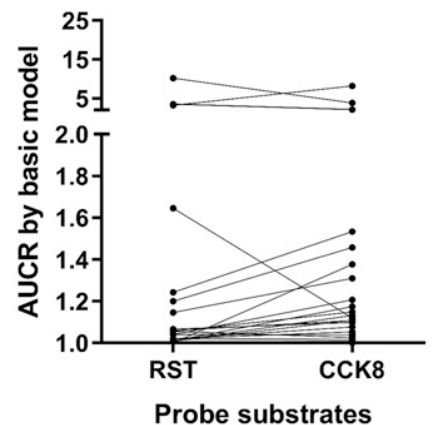

Fig. 1. Comparison of RST AUCR for 22 drugs calculated by basic model using $\mathrm{IC}_{50}$ values with probe substrates (A) $\mathrm{E}_{2} 17 \beta \mathrm{G}$ vs. RST for OATP1B1 inhibition and (B) CCK8 vs. RST for OATP1B3 in HEK293 cells overexpressing these transporters.

2.10), cyclosporine (10.2 vs. 3.84), and velpatasvir (1.65 vs. 1.12) for CCK-8 versus RST, respectively, for OATP1B3.

Qualitative Cutoff-Based Assessment on DDI Predictions Using the Static Model. The static models used in this analysis took into account the fraction of systemic clearance of RST mediated by OATP1B1/3 alone. With a cutoff of $\geq 1.1$ for predicted AUCR, the PPV and NPV for using either RST or $\mathrm{E}_{2} 17 \beta \mathrm{G} / \mathrm{CCK} 8$ as probe substrates were comparable; the PPV are 0.875 and 0.889 for RST and $\mathrm{E}_{2} 17 \beta \mathrm{G} / \mathrm{CCK} 8$, respectively (Supplemental Table 2; Table 1). NPV was 0.692 when RST was used as probe, whereas that with $\mathrm{E}_{2} 17 \beta \mathrm{G} / \mathrm{CCK} 8$ was 0.750 . The cutoff of $\geq 1.04$ for predicted AUCR was also assessed for the predictive performance (Table 1). When only OATP1B1/3 inhibition was considered, the PPVs for RST and $\mathrm{E}_{2} 17 \beta \mathrm{G} / \mathrm{CCK} 8$ were lower than those using the cutoff of $\geq 1.1$. NPV increased to 0.857 and 0.714 , respectively, for RST and $E_{2} 17 \beta \mathrm{G} / \mathrm{CCK} 8$.

Qualitative Cutoff-Based Assessment on DDI Predictions Using the Combined Static Model. The combined static model used in this analysis took into account the fraction of systemic clearance of RST mediated by OATP1B1/3 together with gut BCRP for drugs with $\mathrm{I}_{\text {gut }}$ / $\mathrm{IC}_{50} \geq 10$ for BCRP inhibition. When both OATP1B1/3 and gut BCRP inhibitions were considered, the PPV was 0.688 and 0.667 (for RST and $\mathrm{E}_{2} 17 \beta \mathrm{G} / \mathrm{CCK} 8$, respectively), and the number of $\mathrm{FN}$ prediction improved, with NPV being 1 (i.e., no false negatives) for RST and 0.833 for $\mathrm{E}_{2} 17 \beta \mathrm{G} / \mathrm{CCK} 8$ (Fig. 2; Supplemental Table 2; Table 1). With a cutoff value of 1.04, PPVs were slightly lower than that using the cutoff of $\geq 1.1$ ( 0.647 and 0.556 for RST and $\mathrm{E}_{2} 17 \beta \mathrm{G} / \mathrm{CCK} 8$, respectively). For RST, the NPV was the same as that using the cutoff of $\geq 1.1$, and for $E_{2} 17 \beta \mathrm{G} / \mathrm{CCK} 8$ the NPV was 0.667 .

Quantitative Assessment on DDI Predictions Using the Static Model and the Combined Static Model. The RMSEs of predictions using the static model were 1.31 and 1.24 for RST and $\mathrm{E}_{2} 17 \beta \mathrm{G} / \mathrm{CCK} 8$, respectively (Fig. 2; Supplemental Table 2; Table 1). The RMSEs of predictions using the combined static model when intestinal BCRP inhibition was also taken into account in addition to OATP1B1/3 inhibition were 0.767 and 0.812 for RST and $E_{2} 17 \beta \mathrm{G} / \mathrm{CCK} 8$, respectively (Fig. 2; Supplemental Table 2; Table 1).

DDI Simulations between RST and Rifampicin, Velpatasvir, and Asunaprevir Using PBPK Modeling. PBPK models were constructed to retrospectively predict DDI of rifampicin, asunaprevir, and velpatasvir with RST. To sufficiently describe the observed AUCR, a scaling factor was applied to the experimental $\mathrm{IC}_{50}$ before incorporating into the PBPK model. This scaling factor was found to be substrate-dependent; for $\mathrm{IC}_{50}$ obtained from inhibitory assays with RST as substrate, a factor of 200 was applied, and a factor of 100 was applied to those obtained from assay using $\mathrm{E}_{2} 17 \beta \mathrm{G} / \mathrm{CCK} 8$ as substrates (Supplemental Table 4). Similar scaling factors were applied in other reported studies (Chen et al., 2018; Yoshida et al., 2018). The PBPK models reasonably predicted the magnitude of DDI between RST and rifampicin and asunaprevir with the predicted AUCR $<1.5$-fold difference compared with the observed AUCR (Fig. 3) (Prueksaritanont et al., 2014, 2017; Eley et al., 2015; Lai et al., 2016; Shen et al., 2017; Takehara et al., 2018). The DDI simulation between RST and velpatasvir was underpredicted with a 2-fold difference compared with the observed AUCR (Mogalian et al., 2016).

\section{Discussion}

In this study, we evaluated the suitability of AUCR (predicted AUC ratio due to DDI) cutoff values suggested by the regulatory agencies with the aim of calibrating our internal in vitro assay system for OATP1B1/ 1B3 inhibition for qualitative as well as quantitative DDI predictions (https://www.ema.europa.eu/documents/scientific-guideline/guidelineinvestigation-drug-interactions_en.pdf; https:/www.pmda.go.jp/files/ 000228122.pdf; https://www.fda.gov/media/134582/download). Using either RST or $\mathrm{E}_{2} 17 \beta \mathrm{G} / \mathrm{CCK} 8$ as probe substrates, the in vitro DDI potential of 22 selected drugs, which have previously been studied in clinical DDI studies with RST, was evaluated using the basic model, and the predicted DDI magnitude was compared with the observed DDI in vivo. Substrate-dependent inhibition of OATP1B1/1B3 is well documented (Izumi et al., 2015), and we corroborated the observation that the in vitro assays using $\mathrm{E}_{2} 17 \beta \mathrm{G} / \mathrm{CCK} 8$ as probes yield higher $R$ values (more potent $\mathrm{IC}_{50}$ values) than those using $\mathrm{RST}$ as a probe substrate (Izumi et al., 2015).

For a qualitative analysis, a true or FP or FN prediction was made using the $\mathrm{IC}_{50}$ values to predict magnitude of in vivo DDI for RST using static models (https://www.ema.europa.eu/documents/scientific-guideline/ guideline-investigation-drug-interactions_en.pdf; https://www.pmda.go.jp/ files/000228122.pdf; https://www.fda.gov/media/134582/download). In the context of cutoff values in regulatory guidances, a model-predicted 1.1- or 1.04-fold increase ( $R$ value) in probe substrate AUC was compared with AUC increase observed in vivo, with $\geq 1.25$-fold reported AUC increase considered as a true positive DDI outcome in vivo (https://www. ema.europa.eu/documents/scientific-guideline/guideline-investigationdrug-interactions_en.pdf; https://www.pmda.go.jp/files/000228122.pdf; https://www.fda.gov/media/134582/download). When clinical relevance of these AUCR predictions was considered, the NPV and PPV of these predictions using static model were comparable for all the probe substrates tested.

To translate the inhibitory potency from in vitro inhibition assays to more accurate quantitative prediction, approaches with mechanistic understanding are needed, such as a static mechanistic model that combines inhibition of multiple clearance pathways or even a more comprehensive, dynamic PBPK model. To use these models, a thorough understanding of the disposition pathway of the clinical probe substrate (i.e., RST) is essential. For instance, although OATP1B1 and OATP1B3 are identified as the key players in the hepatic uptake of RST, the involvement of the BCRP also needs careful consideration because it governs the absorption of the RST from the intestine (Elsby et al., 2012, 2016; Hua et al., 2012; Bae et al., 2018). Studies have found BCRP is responsible for as much as $50 \%$ of drug efflux from gut enterocyte. Similarly, NTCP also participates in the transport of RST in the liver. Based on in vitro data, the relative contributions of OATP1B1, OATP1B3, and NTCP to the overall hepatic uptake of RST have been estimated to be $70 \%, 20 \%$, and 10\%, respectively (Wang et al., 2017). The hepatic clearance of RST was reported to be approximately $72 \%$ of 
TABLE

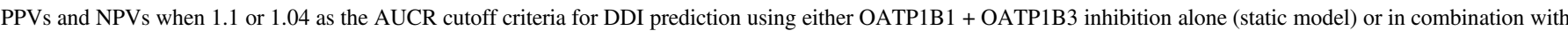
BCRP inhibition (combined static model)

Either E217 $3 / \mathrm{CCK} 8$ or RST were used as in vitro probes for OATP1B inhibition.

\begin{tabular}{|c|c|c|c|c|c|c|c|c|}
\hline \multirow{3}{*}{$\frac{\text { Cutoff criteria }}{\text { In vitro substrate }}$} & \multicolumn{4}{|c|}{ AUCR (Only OATP1B1 and OATP1B3) } & \multicolumn{4}{|c|}{ AUCR (OATP1B1, OATP1B3, and BCRP for $\left.\mathrm{I}_{\mathrm{gut}} / \mathrm{IC}_{50}>10\right)$} \\
\hline & \multicolumn{2}{|c|}{$\mathrm{AUCR} \geq 1.04$} & \multicolumn{2}{|c|}{$\mathrm{AUCR} \geq 1.1$} & \multicolumn{2}{|c|}{$\mathrm{AUCR} \geq 1.04$} & \multicolumn{2}{|c|}{$\mathrm{AUCR} \geq 1.1$} \\
\hline & RST & $\mathrm{E}_{2} 17 \beta \mathrm{G} / \mathrm{CCK} 8$ & RST & $\mathrm{E}_{2} 17 \beta \mathrm{G} / \mathrm{CCK} 8$ & RST & $\mathrm{E}_{2} 17 \beta \mathrm{G} / \mathrm{CCK} 8$ & RST & $\mathrm{E}_{2} 17 \beta \mathrm{G} / \mathrm{CCK} 8$ \\
\hline PPV & 0.714 & 0.643 & 0.875 & 0.889 & 0.647 & 0.556 & 0.688 & 0.667 \\
\hline Number of TP/number of TP + FP & $10 / 14$ & $9 / 14$ & $7 / 8$ & $8 / 9$ & $11 / 17$ & $10 / 18$ & $11 / 16$ & $10 / 15$ \\
\hline NPV & 0.857 & 0.714 & 0.692 & 0.750 & 1 & 0.667 & 1 & 0.833 \\
\hline Number of TN/number of $\mathrm{TN}+\mathrm{FN}$ & $6 / 7$ & $5 / 7$ & $9 / 13$ & $9 / 12$ & $4 / 4$ & $2 / 3$ & $5 / 5$ & $5 / 6$ \\
\hline RMSE & \multicolumn{4}{|c|}{1.31 (RST as probe); 1.24 ( $\mathrm{E}_{2} 17 \beta \mathrm{G} / \mathrm{CCK} 8$ as probes) } & \multicolumn{4}{|c|}{0.767 (RST as probe); $0.812\left(\mathrm{E}_{2} 17 \beta \mathrm{G} / \mathrm{CCK} 8\right.$ as probes) } \\
\hline
\end{tabular}

the total plasma clearance (Martin et al., 2003a,b). Hence, the fractional contribution of transporters toward the clearance of RST can be estimated to be $0.504,0.144$, and 0.072 for OATP1B1, OATP1B3, and NTCP, respectively. Other studies report somewhat higher contribution of OATP1B3, OATP2B1 and NTCP at 10\%-35\% toward RST clearance (Ho et al., 2006; Bi et al., 2013; Wang et al., 2017; Zhang et al., 2019). Given the difficulties in accurate estimation of contribution by each transporter in RST clearance, in this study we considered only the roles of BCRP and OATP1B1/3 in RST disposition, since together these account for a majority of RST clearance. In addition, our focus was to determine the most reliable evaluation of in vitro data requested by regulatory agencies to better predict RST DDI. This allowed for a reasonable prediction of the AUCR while maintaining a simple model.

Generally, there is also a concern about accurate DDI prediction for highly protein-bound drugs ( $>99 \%$ ) for OATP1B1/1B3. In our assessments, almost all the drugs reported in the University of Washington DDI Database, including the ones used in our study, which had both a DDI with rosuvastatin (in vivo AUCR $>1.25$ ) and were highly protein-bound $(>99 \%$ bound), also inhibited BCRP in vitro (list provided in Supplemental Table 6). Since prediction of gut BCRP inhibition takes into account the total gut concentrations of the inhibitor,
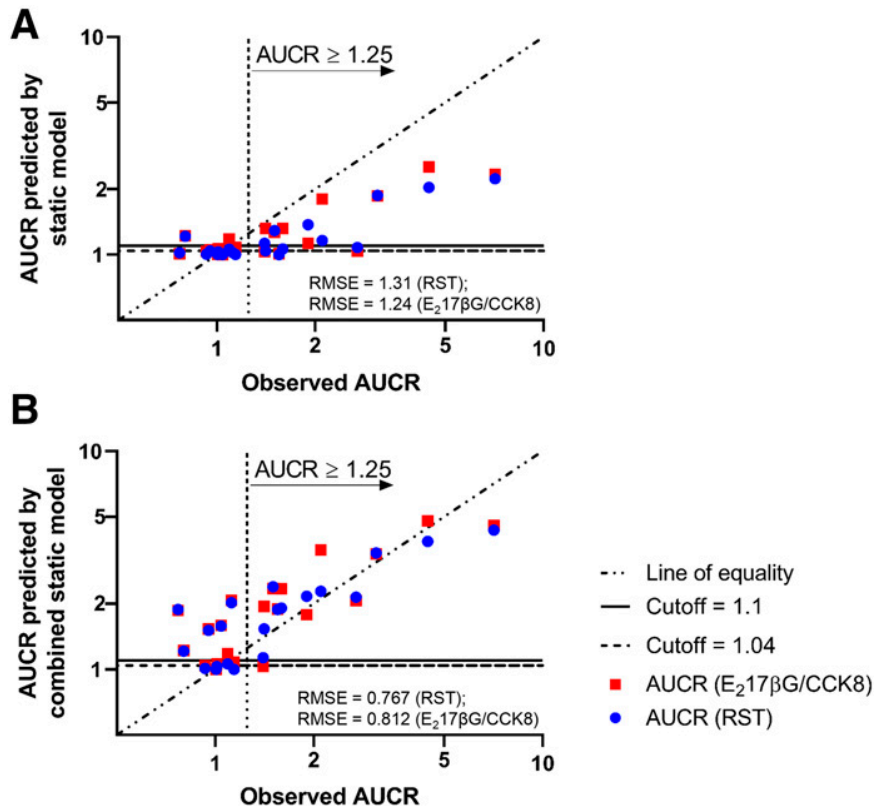

Fig. 2. Quantitative predictions of AUCR for RST mediated by 22 studied drugs using (A) static model, which only considered OATP1B $1 / 3$ inhibition, and (B) combined static model, which took into account intestinal BCRP inhibition in addition to OATP1B1/3 inhibition. the impact of protein-binding value used in overall predictions for these drugs (i.e., actual $<1 \%$ value vs. rounded up to $1 \%$ unbound as recommended in the regulatory guidances) did not significantly impact the predictions.

Using DDI guidance cutoff values, the two static models were compared for their qualitative predictive performance. For a quantitative analysis, the magnitude of the predicted DDI using static model of OATP1B1/1B3 inhibition was modified to incorporate the gut BCRP inhibition, and the predictions with the combined static model were comparatively better than with the static model using OATP1B1/1B3 inhibition alone.

From a qualitative point of view, as described before, despite the substrate-dependent differences in the $\mathrm{IC}_{50}$ potencies for either RST or $\mathrm{E}_{2} 17 \beta \mathrm{G} / \mathrm{CCK} 8$ as in vitro probe substrates, the PPV and NPV metrics were fairly comparable when only OATP1B1 and OATP1B3 were considered as clearance mechanisms for RST. Although PPV and NPV were high, there were four FNs and one FP. However, it is noteworthy that except for velpatasvir, which has a complex disposition, all the other FNs appeared to cause only a mild DDI with $<1$.6-fold increase in RST AUC observed in vivo (Mogalian et al., 2017). Therefore, this calibration analysis suggests that the cutoff of 1.1 is reasonable and adequate for the in vitro systems tested here to capture clinically meaningful DDI of $\geq 1$. 6 -fold.

From a quantitative point of view, the static model that only considers OATP1B1/1B3 slightly underpredicted the magnitude of the interaction (Fig. 2A). Inclusion of BCRP inhibition in both static mechanistic model

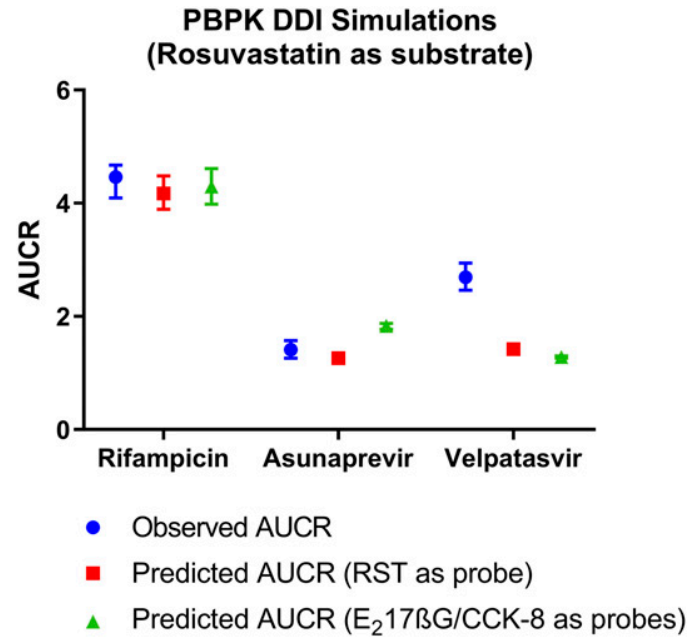

Fig. 3. DDI simulations between RST and rifampicin, velpatasvir, and asunaprevir using PBPK modeling. For rifampicin, the error bars represent the range of AUCRs reported from three studies (Prueksaritanont et al., 2014; Lai et al., 2016; Shen et al., 2017). For asuanprevir and velpatasvir, the error bars represent the $90 \%$ confidence interval as reported in the respective studies (Eley et al., 2015; Mogalian et al., 2016). 
and PBPK for DDI prediction is crucial for some inhibitors that may inhibit BCRP in addition to OATP1B1/1B3. We used the criteria recommended by regulatory agencies to estimate whether an investigational drug has a potential to inhibit gut BCRP efflux of RST: $\mathrm{I}_{\text {gut }} / \mathrm{IC}_{50} \geq 10$, in which $\mathrm{I}_{\text {gut }}=$ dose of inhibitor/250 ml (https:/www.ema.europa.eu/documents/ scientific-guideline/guideline-investigation-drug-interactions_en.pdf; https://www.pmda.go.jp/files/000228122.pdf; https://www.fda.gov/ media/134582/download). With the combined static model, which incorporated both inhibition of OATP1B1/1B3 and BCRP when relevant, the quantitative prediction improved as compared with OATP1B1/1B3 alone (Fig. 2B). The success of this approach for a broad range of compounds studied here corroborates the understanding that gut BCRP plays a significant role in RST disposition (Elsby et al., 2012, 2016). The qualitative metric NPV improved with this model with no FNs, but the PPV decreased slightly because of an increase in FPs (Supplemental Table 2). Two potential reasons could be the overestimation of the in vitro inhibitory potential in vesicular transport assay because of the lack of protein binding as well as easier access to transporter in inside-out vesicles and overprediction of the drug concentration in the gut enterocytes leading to overestimation of DDI at the enterocyte level in this empirical, static approach. As such, the in vitro to in vivo translation of gut BCRP inhibition from in vitro assays may need further tuning to reduce the FP cases.

To overcome the limitation of empirical estimation of static gut and liver concentrations, PBPK modeling was conducted for 3 of the 22 drugs tested. This dynamic mechanistic approach retrospectively complemented the findings of the static model for DDI. PBPK models reasonably predicted the DDI observed with rifampicin and asunaprevir. The model underpredicted the DDI with velpatasvir, however, within 2-fold of the observed AUCR. Velpatasvir is a Biopharmaceutics Classification System class IV drug with low permeability and low solubility, making it challenging to predict the enterocyte concentration, which may result in underprediction of DDI (https://www.ema.europa.eu/en/documents/assessment-report/ epclusa-epar-public-assessment-report_en.pdf). Substrate-specific scaling factors, which are in line with those used in Wang et al. (2017), were used in this study to describe the clinical PK. These scaling factors can be applied to other drugs and their in vitro $\mathrm{IC}_{50}$ values obtained using the same experimental system for more reliable predictions using PBPK modeling in the future. This approach is particularly useful for circumstances that the static model cannot incorporate, such as physiologic changes due to disease state and physiologic differences.

Using the approach described herein, we retrospectively predicted the magnitude of DDI for fenebrutinib and compared it with the recently reported clinical DDI data with RST, wherein a 2.63-fold increase in AUC was seen for RST when coadministered with fenebrutinib (Jones et al., 2020). The predicted AUCRs using the static model for OATP1B1 and OATP1B3 are 1.2 and 1.5 , respectively. Since the $\mathrm{I}_{\text {gut }} / \mathrm{IC}_{50}$ for BCRP was 128 - that is, greater than 10-incorporation of BCRP is important for more accurate DDI prediction for fenebrutinib. By using the combined static model incorporating both OATP1B and BCRP, the AUCR was predicted to be 2.15, which is closer to the observed AUCR than with using OATP1B1/1B3 alone (Jones et al., 2020). This example illustrates the advantage of using combined static model to provide a more accurate quantitative prediction. Such an approach can be employed to systematically investigate and then predict DDI involving multiple transporters with other probes of interest, such as pitavastatin or pravastatin.

Overall, our results highlighted that: 1) from a qualitative perspective, the current $R$ value cutoff criteria recommended by the U.S. Food and
Drug Administration and the Pharmaceuticals and Medical Devices Agency in Japan $(R>1.1)$ appears to be reasonable for the in vitro system tested in our laboratories to capture potential to cause a clinically relevant DDI, and 2) from a quantitative perspective, combined static model that takes into OATP1B1/1B3 and BCRP inhibition by perpetrators provides a more mechanistic and accurate approach to predict extent of transporter-mediated DDI with RST. Dynamic modeling also performed reasonably well and has greater utility in cases wherein static models are inadequate to capture the overall DDI liability.

\section{Authorship Contributions}

Participated in research design: Sane, Cheung, Kovács, Farasyn, Gáborik.

Conducted experiments: Sane, Cheung, Kovács, Farasyn, Li, Bui, Gáborik. Contributed new reagents or analytic tools: Kovács, Li, Bui, Kis, Plise, Gáborik.

Performed data analysis: Sane, Cheung, Kovács, Farasyn, Li, Bui, Plise, Gáborik.

Wrote or contributed to the writing of the manuscript: Sane, Cheung, Kovács, Farasyn, Li, Bui, Musib, Kis, Plise, Gáborik.

\section{References}

Bae SH, Park WS, Han S, Park GJ, Lee J, Hong T, Jeon S, and Yim DS (2018) Physiologicallybased pharmacokinetic predictions of intestinal BCRP-mediated drug interactions of rosuvastatin in Koreans. Korean J Physiol Pharmacol 22:321-329.

Bi YA, Qiu X, Rotter CJ, Kimoto E, Piotrowski M, Varma MV, Ei-Kattan AF, and Lai Y (2013) Quantitative assessment of the contribution of sodium-dependent taurocholate co-transporting polypeptide (NTCP) to the hepatic uptake of rosuvastatin, pitavastatin and fluvastatin. Biopharm Drug Dispos 34:452-461.

Chen Y, Zhu R, Ma F, Mao J, Chen EC, Choo EF, Sahasranaman S, and Liu L (2018) Assessment of OATP transporter-mediated drug-drug interaction using physiologicallybased pharmacokinetic (PBPK) modeling - a case example. Biopharm Drug Dispos 39: $420-430$.

Eley T, Han YH, Huang SP, He B, Li W, Bedford W, Stonier M, Gardiner D, Sims K, Rodrigues $\mathrm{AD}$, et al. (2015) Organic anion transporting polypeptide-mediated transport of, and inhibition by, asunaprevir, an inhibitor of hepatitis C virus NS3 protease. Clin Pharmacol Ther 97: $159-166$.

Elsby R, Hilgendorf C, and Fenner K (2012) Understanding the critical disposition pathways of statins to assess drug-drug interaction risk during drug development: it's not just about OATP1B1. Clin Pharmacol Ther 92:584-598.

Elsby R, Martin P, Surry D, Sharma P, and Fenner K (2016) Solitary inhibition of the breast cancer resistance protein efflux transporter results in a clinically significant drug-drug interaction with rosuvastatin by causing up to a 2 -fold increase in statin exposure. Drug Metab Dispos 44: rosuvastatin 408 .

Heredi-Szabo K, Kis E, and Krajcsi P (2012) The vesicular transport assay: validated in vitro methods to study drug-mediated inhibition of canalicular efflux transporters ABCB11/BSEP and ABCC2/MRP2. Curr Protoc Toxicol Chapter 23:Unit 23.4.

Ho RH, Tirona RG, Leake BF, Glaeser H, Lee W, Lemke CJ, Wang Y, and Kim RB (2006) Drug and bile acid transporters in rosuvastatin hepatic uptake: function, expression, and pharmacogenetics. Gastroenterology 130:1793-1806.

Hua WJ, Hua WX, and Fang HJ (2012) The role of OATP1B1 and BCRP in pharmacokinetics and DDI of novel statins. Cardiovasc Ther 30:e234-e241.

Izumi S, Nozaki Y, Maeda K, Komori T, Takenaka O, Kusuhara H, and Sugiyama Y (2015) Investigation of the impact of substrate selection on in vitro organic anion transporting polypeptide 1B1 inhibition profiles for the prediction of drug-drug interactions. Drug Metab Dispos 43:235-247.

Jones NS, Yoshida K, Salphati L, Kenny JR, Durk MR, and Chinn LW (2020) Complex DDI by fenebrutinib and the use of transporter endogenous biomarkers to elucidate the mechanism of DDI. Clin Pharmacol Ther 107:269-277.

Lai Y, Mandlekar S, Shen H, Holenarsipur VK, Langish R, Rajanna P, Murugesan S, Gaud N, Selvam S, Date O, et al. (2016) Coproporphyrins in plasma and urine can be appropriate clinical biomarkers to recapitulate drug-drug interactions mediated by organic anion transporting polypeptide inhibition. J Pharmacol Exp Ther 358:397-404.

Martin PD, Warwick MJ, Dane AL, Brindley C, and Short T (2003a) Absolute oral bioavailability of rosuvastatin in healthy white adult male volunteers. Clin Ther 25:2553-2563.

Martin PD, Warwick MJ, Dane AL, Hill SJ, Giles PB, Phillips PJ, and Lenz E (2003b) Metabolism, excretion, and pharmacokinetics of rosuvastatin in healthy adult male volunteers. Clin Ther 25: 2822-2835.

Mogalian E, German P, Kearney BP, Yang CY, Brainard D, Link J, McNally J, Han L, Ling J, and Mathias A (2017) Preclinical pharmacokinetics and first-in-human pharmacokinetics, safety, and tolerability of velpatasvir, a pangenotypic hepatitis C virus NS5A inhibitor, in healthy subjects. Antimicrob Agents Chemother 61:e02084-16.

Mogalian E, German P, Kearney BP, Yang CY, Brainard D, McNally J, Moorehead L, and Mathias A (2016) Use of multiple probes to assess transporter- and cytochrome P450-mediated drug-drug interaction potential of the pangenotypic HCV NS5A inhibitor velpatasvir. Clin Pharmacokinet 55:605-613.

Prueksaritanont T, Chu X, Evers R, Klopfer SO, Caro L, Kothare PA, Dempsey C, Rasmussen S, Houle R, Chan G, et al. (2014) Pitavastatin is a more sensitive and selective organic anion-transporting polypeptide $1 \mathrm{~B}$ clinical probe than rosuvastatin. Br J Clin Pharmacol 78:587-598. 
Prueksaritanont T, Tatosian DA, Chu X, Railkar R, Evers R, Chavez-Eng C, Lutz R, Zeng W, Yabut J, Chan GH, et al. (2017) Validation of a microdose probe drug cocktail for clinical drug interaction assessments for drug transporters and CYP3A. Clin Pharmacol Ther 101:519-530.

Shen H, Chen W, Drexler DM, Mandlekar S, Holenarsipur VK, Shields EE, Langish R, Sidik K, Gan J, Humphreys WG, et al. (2017) Comparative evaluation of plasma bile acids, dehydroepiandrosterone sulfate, hexadecanedioate, and tetradecanedioate with coproporphyrins I and III as markers of OATP inhibition in healthy subjects. Drug Metab Dispos 45:908-919.

Takehara I, Yoshikado T, Ishigame K, Mori D, Furihata KI, Watanabe N, Ando O, Maeda K, Sugiyama Y, and Kusuhara H (2018) Comparative study of the dose-dependence of OATP1B inhibition by rifampicin using probe drugs and endogenous substrates in healthy volunteers. Pharm Res 35:138.

Vaidyanathan J, Yoshida K, Arya V, and Zhang L (2016) Comparing various in vitro prediction criteria to assess the potential of a new molecular entity to inhibit organic anion transporting polypeptide 1B1. J Clin Pharmacol 56 (Suppl 7):S59-S72.

Wang Q, Zheng M, and Leil T (2017) Investigating transporter-mediated drug-drug interactions using a physiologically based pharmacokinetic model of rosuvastatin. CPT Pharmacometrics Syst Pharmacol 6:228-238.
Yoshida K, Guo C, and Sane R (2018) Quantitative prediction of OATP-mediated drug-drug interactions with model-based analysis of endogenous biomarker kinetics. CPT Pharmacometrics Syst Pharmacol 7:517-524.

Yoshida K, Maeda K, and Sugiyama Y (2012) Transporter-mediated drug--drug interactions involving OATP substrates: predictions based on in vitro inhibition studies. Clin Pharmacol Ther 91:1053-1064.

Zhang Y, Panfen E, Fancher M, Sinz M, Marathe P, and Shen H (2019) Dissecting the contribution of OATP1B1 to hepatic uptake of statins using the OATP1B1 selective inhibitor estropipate. Mol Pharm 16:2342-2353.

Address correspondence to: Dr. Rucha Sane, Department of Clinical Pharmacology, Genentech, Inc., 1 DNA Way, South San Francisco, CA 94080. E-mail: sane.rucha@gene.com; or Dr. Zsuzsanna Gáborik, SOLVO Biotechnology, Irinyi Jozsef u 4-20, Budapest 1117, Hungary. E-mail: zsuzsanna.gaborik@crl.com 